\title{
FACTORES QUE INFLUYEN EN LA SELECCIÓN DEL CONCESIONARIO EN LA COMPRA DE MOTOCICLETAS EN MEDELLÍN: ESTUDIO DE CASO
}

\author{
Factors affecting the selection of dealer in the purchase of motorcycles in Medellin: a case study
}

\author{
Juan Carlos Pérez Vélez
}

Estudiante de ingeniería industrial. Universidad Nacional de Colombia, Medellín - Colombia, jucperezve@unal.edu.co

\section{Hervin Smiller Zuluaga Sepúlveda}

Estudiante de ingeniería industrial. Universidad Nacional de Colombia, Medellín - Colombia, hszuluagas@unal.edu.co

\section{Juan Diego Orozco Galeano}

Estudiante de ingeniería industrial. Universidad Nacional de Colombia, Medellín - Colombia, jdorozcog@unal.edu.co

\section{Paulo Andrés González Almeyda}

Estudiante de ingeniería Mecánica. Universidad Nacional de Colombia, Medellín - Colombia, agonzaleza@unal.edu.co

\section{Cómo citar/ How to cite}

Pérez, J., Zuluaga, H., Orozco, J. y González, P. (2015). Factores que influyen en la selección del concesionario en la compra de motocicletas en Medellín: Estudio de caso. Revista CEA, 1(2), 63-77.

Recibido: 29 de enero de 2015

Aceptado: 12 de marzo de 2015

\section{Resumen}

Este estudio se realizó con el fin de establecer cuáles son los criterios que el consumidor tiene en consideración al momento de seleccionar el concesionario para la compra de motocicletas. En este sentido, la recolección de información se hizo teniendo en cuenta únicamente sujetos que hubieran adquirido motocicletas en concesionario. Asumiendo una investigación de tipo cuantitativo, una muestra teórica de 294 individuos y una participación real de 60 individuos seleccionados de forma probabilística, se midió la influencia de diversos factores relacionados con la decisión de compra. Como resultado de esta medición, y en concordancia con la bibliografía consultada, se encontró que uno de los factores que los sujetos encuestados consideraron como más determinantes para elegir en dónde comprar su motocicleta es la influencia de las recomendaciones de terceros, y entre las principales conclusiones a las que se llegó es que los obsequios entregados por la compra no necesariamente aumentan el nivel de ventas, por lo cual, ofrecerlos a aquellos compradores que lleven referidos o invertir en mejorar la calidad del servicio podría ser una buena alternativa para aprovechar los factores más significativos.

Palabras clave: valor agregado, obsequios, motocicletas, decisión de compra.

\begin{abstract}
This study was conducted to establish what are the criteria that the consumer takes into account when selecting the site (licensee) purchase of motorcycles distributed. In this regard, data collection was done considering only subjects who acquired motorcycle dealer. Assuming a quantitative research, a theoretical sample of 294 and a real participation of 60 individuals selected probabilistically, the influence of various factors related to the purchase decision was measured. As a result of this measurement, and consistent with the literature, we found that one of the factors that respondents considered as most crucial subjects to choose where to buy your motorcycle is the influence of the recommendations of others. Among the main conclusions that were reached is that the gifts given by the purchase does not necessarily increase the level of sales, instead offer them to those buyers with referrals or invest in improving the quality of service could be good alternatives to leverage factors rated as the most significant.
\end{abstract}

Keywords: value added, gifts, motorcycles, purchase decision. 


\section{INTRODUCCIÓN}

Las estrategias de marketing han sido utilizadas a fin de lograr un rendimiento superior en el mercado. Las empresas deben trazarse dos objetivos principales para lograr su proyección como empresas: una posición atractiva en el mercado y contar con los recursos necesarios para que se dificulte ser imitadas (Zahay \& Griffin, 2010). Una de las estrategias empresariales para llegar al cumplimiento de dichos objetivos es la implementación de la mezcla de marketing (precio, plaza, producto y promoción) (McDaniel \& Gates, 2007).

En algunos casos, la mezcla de mercadeo se reduce a solo servicio al cliente, plaza y producto, como en el caso de los concesionarios de vehículos de dos ruedas en donde se deben contrarrestar dos tipos de problemáticas en su proceso de venta del producto al consumidor: la competencia entre productos de diferentes marcas y la competencia entre concesionarios de la misma marca. En este sentido, la decisión del cliente puede estar determinada por el servicio al cliente en las etapas de venta y postventa del producto, y en ciertos casos por la ubicación del concesionario (Kathiravanaa, Panchanathamaa, \& Anushan, 2010).

Para poder llegar a una solución a este tipo de problemas es muy común que estos sean abordados por medio de técnicas de percepción de satisfacción del consumidor, en las que se ven diferentes diseños de cuestionarios (cualitativos y cuantitativos) y elaboración de métricas, a través de las cuales se trata de llegar a un conocimiento de lo que el consumidor busca en el momento de tomar su decisión de compra (Zahay \& Griffin, 2010).

En este artículo se lleva a cabo el análisis cuantitativo de una investigación de mercados realizada mediante la aplicación de una encuesta, con el fin de identificar los factores más influyentes en la selección del lugar para realizar la compra de motocicletas.
Uno de los principales hallazgos de la investigación presentada es la importancia que el cliente le da a la calidad de la atención, por encima de otros factores tales como la oferta de obsequios, y se evidenció, además, que pueden existir carencias en las estrategias de atención al cliente, puesto que la calidad de esta no es altamente influyente en la decisión de compra, aun cuando los clientes consideran que debería serlo. También fue posible validar el planteamiento de algunos autores que tratan la gran influencia de terceros (amigos, familiares, y demás personas allegadas al cliente) en la decisión de compra de motocicletas.

\section{MARCO TEÓRICO}

\section{Contextualización del problema}

Mientras que en términos generales el crecimiento industrial en Colombia parece ir disminuyendo, el sector de los automotores se destaca por estar entre aquellos que lograron crecimiento a enero de 2013 (Ministerio de Comercio Industria y Turismo de Colombia, 2013). La Dirección de Impuestos y Aduanas Nacionales DIAN- indicó que entre los años 2011 y 2012 hubo un incremento por encima del $9.5 \%$ en la producción nacional de motocicletas, pasando de 520.189 a 569.636 motocicletas nuevas producidas, y ubicando al país en el segundo lugar de América Latina en producción de motocicletas, luego de Brasil (Comité de Ensambladoras de Motos Japonesas, 2013). La ANDI, citando cifras del RUNT (Registro Único Nacional de Tránsito) menciona un crecimiento en ventas de hasta $10,9 \%$, y por su parte, Fenalco Antioquia en su Boletín Sectorial Motos N 19 de 2013 dice que en Colombia el $16,5 \%$ de la población ocupada se desplaza a sus lugares de trabajo en moto y hasta un $20,6 \%$ de los hogares tienen motocicleta (Fenalco Antioquia, 2013).

Esta dinámica de crecimiento implica nuevos retos para las empresas, de modo que un gran número de usuarios de motocicletas hace que las compañías y ensambladoras se vean en la necesidad de analizar el comportamiento de 
compra de sus consumidores, lo cual ayuda a las compañías a que puedan tomar buenas decisiones sobre el tipo de motocicletas que deben producir. Asimismo, las compañías de motocicletas que tienen algún conocimiento sobre sus clientes van un paso más allá de sus competidores, y más cuando se está en una industria de alto crecimiento (Ngantung, 2013).

Varios autores han hecho esfuerzos para la formación de un cuerpo conceptual que permita el entendimiento de la conducta de los compradores de motocicletas. Entre ellos se encuentra Solomon (2011), quien plantea que el comportamiento del consumidor abarca un gran terreno y lo define como «el estudio de los procesos involucrados cuando un individuo o grupo elige, compra, usa o dispone de productos, servicios, ideas o experiencias para satisfacer sus necesidades y deseos». Por su parte, Laudon \& Traver (2011) afirman que "el comportamiento del consumidor es una disciplina social que busca modelar y entender el comportamiento de las personas en un mercado», mientras que, según Pride et al. (2010), la conducta de compra del consumidor se refiere a la adquisición de productos personales o de uso doméstico, sin fines de negocio.

Para llegar a un conocimiento del comportamiento del consumidor, algunas investigaciones hablan de cuatro características generales que son claves por la influencia que tienen en el comportamiento de compra de los consumidores: de tipo cultural, social, personal y psicológico (Reddy, 2011). Si bien algunos de estos factores no pueden ser afectados por el mercadeo, sí pueden ser muy útiles en la identificación de compradores potenciales y en la conformación de productos y servicios que se adapten mejor a las necesidades del consumidor. Además, la combinación de estas características forman una actitud de consumo que determina la demanda de los productos y la forma como los bienes y servicios existentes se venden (Srinivas, 2011).

El comportamiento del consumidor se ve afectado por una serie de variables, entre las que se encuentran: necesidades profesionales $y$ personales, actitudes y valores, características de personalidad, sociales, económicas, antecedentes culturales, la edad, el género, estatus profesional - las influencias sociales de diversa índole ejercidas por la familia, amigos, colegas, y la sociedad en su conjunto (Reddy, 2011).

Particularmente, respecto a las variables involucradas en las investigaciones de mercado de motocicletas, se ha observado que la decisión del consumidor de comprar una motocicleta se basa en la propia percepción, la experiencia y la creencia, y el papel de amigos, familiares y miembros de la familia es significativo en dicho proceso de compra. La preferencia de la marca, por su parte, es altamente dependiente de la imagen, el kilometraje y el precio (Srinivas, 2011).

También se observa que la publicidad en la televisión, periódicos y revistas puede ser muy eficaz en dar información a los consumidores sobre el vehículo y sus características. Por lo tanto, la decisión de compra de motos no se basa en una sola variable, sino que es una decisión multivariable, y en muchas ocasiones requiere un estudio cuidadoso de las necesidades socioeconómicas de los clientes (Srinivas, 2011).

\section{Proceso de decisión de compra}

En general, el deseo de tener motocicleta surge de la necesidad de transporte (Chadha, 2011). Las personas con poco tiempo buscan la manera de llegar a los lugares que necesitan ágilmente (Ngantung, 2013) y con la creciente sobrepoblación de vehículos, cada vez el mercado de las motocicletas es más atractivo (Lomboan, Saerang, \& Pangemanan, 2013), dado que son una solución a los problemas personales de movilidad en las ciudades. Frente a lo anterior, se ha encontrado que los usuarios de motocicletas acceden a estos productos básicamente por los siguientes motivos: 1) comodidad y conveniencia, 2) opción alternativa de transporte, 3) facilidades de préstamo, 4) símbolo de status social (Chadha, 2011). 
Los expertos en mercadeo han definido que los usuarios de motocicletas no solo adquieren motocicletas por necesidad sino que su decisión se ve afectada por factores de motivación, percepción, creencias y actitudes (Srinivas, 2011). Cuando la necesidad está, se crea una intención de compra, y esta intención de compra representa la posibilidad de que un consumidor esté planeando realizar una adquisición (Wu, Yeong-Yuh Yeh, \& Chieh-Ru, 2011). Frente a esto, es importante contrastar dicho proceso con los del proceso de compra planteado por Lomboan et al. (2013), el cual consiste en los siguientes pasos: 1) reconocimiento del problema, en donde el consumidor toma conciencia de que necesita algo para estar mejor, 2) búsqueda de información, en donde el consumidor se asesora acerca de cómo va a llegar a ese estado deseado, 3) evaluación de las alternativas, en donde el comprador compara las opciones que investigó desde las fuentes de información, 4) se da un proceso cognitivo complejo en donde se ponen a la luz las ventajas y desventajas de cada alternativa para terminar en la elección de una de ellas (Lomboan et al., 2013).

Sin embargo, Chadha (2011) va más allá y dice que la decisión del consumidor no termina con el acto de adquirir el producto, en este caso la motocicleta, sino que continúa con el uso que este le dé y su posterior evaluación. Los compradores juzgan su experiencia comparándola con sus expectativas y compartiéndola con otros (Chadha, 2011).

\section{Opciones metodológicas utilizadas en la investigación del mercado de motocicletas}

Srinivas (2011), en un estudio que realizó para Hero Honda, presenta algunas características metodológicas, como el hecho de la realización de dicha investigación a partir de encuestas estructuradas y dirigidas a clientes de la compañía y no al público en general. También se encuentra que los datos fueron tomados de dos tipos de fuentes: unas primarias, haciendo uso de encuestas estructuradas y otras secundarias, aprovechando registros de la compañía, páginas web y otros (Srinivas, 2011).
En general, los estudios encontrados han sido realizados con base en encuestas estructuradas que luego son analizadas mediante técnicas estadísticas como los modelos de regresión.

Por ejemplo, Lomboan, Saerang, \& Pangemanan (2013), definen como variables independientes la motivación y la percepción, y como variable dependiente la decisión de compra del consumidor. Ellos definen la motivación como los procesos que dan cuenta de la intensidad, dirección y persistencia de una persona en su esfuerzo hacia el logro de una meta; y la percepción, como un proceso mediante el cual los individuos se organizan e interpretan sus impresiones sensoriales con el fin de dar sentido a su entorno (Lomboan et al., 2013).

La percepción también es definida como el proceso por el cual las personas seleccionan, organizan e interpretan las sensaciones, entendiéndose por sensación la respuesta inmediata de los receptores sensoriales (ojos, oídos, nariz, boca, dedos) a estímulos básicos como la luz, el color, el sonido, el olor y la textura (Solomon et al., 2011; citado en Lomboan et al., 2013).

En relación con las variables implicadas, en la investigación realizada por Omesh Chadha (2011), utilizando la técnica de Garret, se bosqueja un plano general de los factores que inciden en la decisión, que presentados en orden de mayor a menor influencia son: eficiencia de combustible, prestigio de la marca, seguridad, colaboración técnica, precio, capacidad del vehículo y términos de garantía. Sin embargo, estudios como el realizado por Ngantung (2013), amplían el espectro de evaluación incluyendo otros factores como publicidad, nombre de marca, calidad y seguridad.

Así Ngantung (2013), en su estudio sobre los atributos de las motocicletas que son determinantes en la compra de estos bienes, luego de definir 6 factores de estudio y un conjunto de variables para cada uno de ellos (para un total de 
20 variables) encontró que 18 de ellas resultaron ser suficientemente significativas, las cuales fueron: servicio al cliente, marca, eficiencia (en la quema de combustible), valor, tiempo de uso (qué tanto es usado o debe ser usado el producto), publicidad, diseño, calidad, variedad, características funcionales, producto local o extranjero, seguridad, precio, credibilidad de la fabricación, ubicación del almacén, experiencias previas, durabilidad y fiabilidad (tiempo que puede durar el vehículo sin haber sufrido ningún tipo de avería). Las dos variables que resultaron ser menos significativas fueron los descuentos sobre el precio y la facilidad de uso del producto.

Adicionalmente, un trabajo llevado a cabo por Srinivas (2011), entre otros detalles, mostró que es muy importante conocer las fuentes de donde los compradores supieron acerca de las motocicletas de la compañía, encontrando en su investigación que el $60 \%$ de los clientes de la compañía conocieron sobre la existencia de las motocicletas Hero Honda por medio del voz a voz, es decir, amigos, parientes o miembros de su familia, y el $40 \%$ restante afirmó que fue por los medios electrónicos de comunicación (Srinivas, 2011).

Esta misma investigación encontró, además, que la decisión de adquirir una motocicleta está muy relacionada con el nivel de ingresos y edad de las personas (personas de mediana edad e ingresos medios, en general). Por su parte, se concluyó que la preferencia de una marca depende mucho de la imagen, el kilometraje y el precio, y finalmente el estudio determinó también que la publicidad debe ser efectiva dando información al consumidor acerca de las características del vehículo (Srinivas, 2011).

El factor psicológico también juega un rol importante en términos de decidir qué motocicleta comprar, ya que la motocicleta es un artículo de alto valor, y por tanto no será fácil para el consumidor decidir cuál es la mejor (Lomboan et al., 2013). En el momento de asesorarse acerca de cuál es la mejor opción, las personas recurren a diversas fuentes de información, siendo las más importantes los amigos (59\%), seguidos de los parientes (23\%) o conocidos, la publicidad (13\%) y por último el consejo del vendedor (5\%) (Chadha, 2011).

En otro estudio independiente, se encontró una proporción similar para los mismos factores, en la cual se tiene que la decisión de compra influenciada por los amigos y conocidos corresponde a un 65\%, la publicidad a un $30 \%$ y vendedores o representantes de la compañía a un 5\% (Yuvaraju, 2014).

\section{PLANTEAMIENTO DEL PROBLEMA}

Con la dinámica de crecimiento del sector automotor, que se viene observando en los últimos años, se hace cada vez más necesario que las compañías y ensambladoras se den a la tarea de analizar el comportamiento de compra de sus consumidores, lo cual ayuda a las organizaciones en la toma de decisiones sobre el tipo de motocicletas que deben producir (Ngantung, 2013), dónde vender, los accesorios a ofrecer o el diseño de promociones que hagan más atractiva la compra. De esta forma, las compañías de motocicletas que tienen algún conocimiento sobre sus clientes aventajan a su competencia, aspecto fundamental cuando se está en una industria de alto crecimiento y con tantos competidores (Ngantung, 2013).

Particularmente en Auteco S.A., se ha observado que uno de los principales criterios que determinan el lugar de compra de una motocicleta es el valor percibido, y entre dos motocicletas de la misma referencia esta diferencia de valor se ve enmarcada por el valor agregado que se ofrezca. Es por esto que una estrategia general en los concesionarios es regalar algún artículo como obsequio por la compra, o en su defecto otorgar un descuento para aumentar el valor que el cliente siente que recibe con la transacción (Chadha, 2011).

Al analizar el problema anteriormente planteado, para el caso particular de Auteco S.A.S (empresa 
dedicada a ensamblar y distribuir motocicletas de cuatro marcas diferentes: KTM, Kawasaki, Bajaj y Kymco), se genera el siguiente interrogante: ¿Cuáles son los criterios que el consumidor tiene en consideración al momento de seleccionar el concesionario para la compra de motocicletas distribuidas por Auteco S.A.S?

\section{METODOLOGÍA DE INVESTIGACIÓN}

Con base en los objetivos de la investigación, se diseñó una encuesta virtual bajo un enfoque cualitativo con el fin de aplicarla a clientes que habían efectuado recientemente la compra de motocicletas en el concesionario "Su moto de la $80 \%$.

La encuesta diseñada consta de 24 preguntas de diferentes tipos (de selección múltiple, dicotómicas, en escala de clasificación Likert, preguntas abiertas y preguntas demográficas), agrupadas en seis factores de interés para la investigación.

Con esta encuesta se pretendía identificar la influencia de diversos factores como la ubicación, la oferta de obsequios, el nivel de servicio o la influencia de terceros sobre la decisión de compra de motocicletas. Además, algunas preguntas de la encuesta estaban encaminadas a conocer el proceso de compra de motocicletas y a hacer una caracterización demográfica de los clientes encuestados.

Con el fin de realizar un muestreo probabilístico y poder inferir sobre toda la población, se seleccionó una muestra aleatoria con base en el listado de los 1.251 clientes registrados en los últimos 6 meses.

El cálculo del tamaño de muestra se realizó con base en la población total de $\mathrm{N}=1.251$ personas, que corresponde a las personas que compraron motocicletas en el concesionario en los últimos 6 meses, considerando un nivel de confianza del 95\% y un error máximo de estimación del 5\% con el fin de obtener datos con una alta confiabilidad, considerando la posibilidad de que puedan ser usados en un futuro para tomar decisiones de marketing.

Como resultado, se obtuvo un tamaño de muestra de 294 personas, la cual fue seleccionada en su totalidad y de manera aleatoria; sin embargo, debido a múltiples dificultades para contactar a los clientes seleccionados, solo fue posible la realización de 60 encuestas.

\section{RESULTADOS Y DISCUSIÓN}

La base de esta investigación fue la aplicación de una encuesta con preguntas elaboradas y agrupadas con el fin de medir los siguientes seis factores:

- Caracterización de la población

- Influencia de la ubicación geográfica

- Influencia del obsequio ofrecido

- Influencia de la calidad del servicio

- Influencia de terceros

- Proceso de compra

El primer factor, (caracterización de la población) está relacionado de forma más indirecta con el objetivo de la investigación, ya que permite establecer un perfil general del tipo de cliente que frecuenta el concesionario, pero no se considera como un factor influyente en la decisión de compra.

El análisis de los siguientes cuatro factores contribuye en gran medida al objetivo de esta investigación, puesto que indica la influencia de cada uno de ellos en la decisión de compra de una motocicleta.

Finalmente, en el factor denominado proceso de compra se busca medir variables como el tiempo promedio de cotización y de búsqueda de la moto y la claridad en el modelo de motocicleta deseado.

\section{Caracterización de la población}

Inicialmente, se caracterizó a la población con el fin de tener una medida de la tipología de clientes, 
con la cual se observó que el perfil de personas que contestaron a la encuesta son sujetos de sexo masculino de mediana edad, pertenecientes a la clase media, con ingresos no superiores a 3 S.M.M.L.V. (Salario Mínimo Mensual Legal Vigente) y que en su mayoría solo han realizado una compra en el concesionario. A continuación se presentan los datos detallados:

- El $77 \%$ de las personas encuestadas son individuos de sexo masculino, el restante 33\% es de sexo femenino.

- El 54\% de las personas encuestadas estaban en edades entre los 16 y los 25 años, el 18\% entre los 25 y 30 años, el 16\% entre los 30 y 35 años, y el $12 \%$ entre los 35 y 40 años de edad.

- El $67 \%$ de encuestados respondieron pertenecer al estrato 3 o 4, seguido por el $28 \%$ que contestaron pertenecer al estrato $1 \circ 2$. Finalmente el 5\% contestó pertenecer al estrato 5 y ninguno de los encuestados afirmó pertenecer al estrato 6 .

- El $85 \%$ de individuos respondió tener un nivel de ingresos entre $\$ 600.000$ y $\$ 1^{\prime} 799.000$, el $10 \%$ entre $\$ 1^{\prime} 800.000$ y $\$ 2^{\prime} 400.000$, y el $5 \%$ contestó que su nivel de ingresos era superior a los $\$ 2^{\prime} 400.000$.

- A la pregunta de si había comprado anteriormente en el concesionario, el 13,33\% de las personas respondieron afirmativamente y el $86,67 \%$ contestaron negativamente a la pregunta.

\section{Análisis por factores}

Para efectuar el respectivo análisis se realizó un compendio de las preguntas principales de cada factor y se acompañó con sus resultados con el fin de hallar patrones relevantes. Asimismo, se observaron las correlaciones existentes entre las diferentes preguntas.
Ubicación

La ubicación hace referencia al nivel de influencia que tuvo en la decisión de compra, la cercanía del concesionario con los lugares que el comprador suele frecuentar.

A la pregunta ¿a cuántas cuadras vive del concesionario? el $21,67 \%$ de los encuestados respondió que a menos de 11 , el $36,67 \%$ contestó que vive entre 11 y 20 cuadras del concesionario, el $31,67 \%$ afirmó vivir entre 21 y 100 cuadras de distancia y el 10\% a más de 100 cuadras del concesionario.

En la Tabla 1 se muestra el promedio de cuadras a las que se ubican las personas del concesionario. Para esto se excluyeron los datos atípicos, los cuales fueron establecidos como aquellos que presentaron un valor mayor a 30, ya que la cercanía a ese nivel no es significativa. En total se excluyeron 17 resultados, y entre los restantes, se obtuvo un promedio de 16 cuadras, con un valor mínimo de 4 cuadras.

Analizando las respuestas de la influencia de la cercanía en la decisión de compra de la moto, se observó que el 58.3\% de los encuestados están de acuerdo en que este factor es, o influyente, o muy influyente. Por otro lado, se encontró que un $31,7 \%$ de los encuestados escogieron el lugar de compra por la cercanía a su casa, el 28,3\% por la cercanía a su zona de trabajo y el $15 \%$ por que queda sobre un camino que frecuentan, haciendo la aclaración de que ninguno de estos porcentajes es excluyente y que no necesariamente su suma debe alcanzar el $100 \%$ de las personas encuestadas, ya que bien pudo suceder que para una persona, el concesionario se encuentre ubicado cerca de estos tres lugares y que esa cercanía influyó sobre la decisión de compra. 
Tabla 1. Compilación de las preguntas de ubicación

Table 1. Compilation of questions location

\begin{tabular}{|c|c|}
\hline $\begin{array}{c}\text { ¿Aproximadamente a cuántas cuadras de su residencia se } \\
\text { ubica el concesionario? }\end{array}$ & 16 cuadras (promedio) \\
\hline \multicolumn{2}{|c|}{ ¿Por cuáles de las siguientes razones escogió el concesionario donde compró su motocicleta? } \\
\hline Cercanía a su casa & $31,70 \%$ \\
\hline Cercanía a su zona de trabajo & $28,30 \%$ \\
\hline Queda sobre un camino que frecuento & $15,00 \%$ \\
\hline \multicolumn{2}{|c|}{ Clasifique la influencia que tuvieron en la compra los siguientes factores: Ubicación } \\
\hline Muy influyente & $15,00 \%$ \\
\hline Influyente & $43,30 \%$ \\
\hline Indiferente & $31,70 \%$ \\
\hline Poco influyente & $5,00 \%$ \\
\hline Muy poco influyente & $5,00 \%$ \\
\hline
\end{tabular}

Fuente: Elaboración propia.

De acuerdo con lo encontrado en el análisis de la Tabla 1, se podría decir que el factor "ubicación» no tiene un efecto fuerte en la decisión del lugar de compra de motocicletas, ya que los porcentajes de influencia y no influencia se encuentran divididos casi que en partes iguales (58.3\% frente a un $41.7 \%$ ), si se considera el ser «indiferente» frente a la ubicación del concesionario en este último grupo.

\section{Obsequios}

El obsequio juega un papel particular en esta situación, debido a que hace parte de un proceso de negociación con el cliente. Con el fin de cerrar la venta, el asesor ofrece al cliente potencial un obsequio a cambio de que él decida comprar allí, entre los que se encuentran la marcación gratuita de la motocicleta, mochilas, gorras, balones, etc. El obsequio se convierte para algunos clientes en un criterio de decisión importante ya que psicológicamente incrementa lo que se conoce como el valor percibido por el cliente y aquellos que le dan importancia a esto van a decidir comprar en un lugar o en otro a partir de los beneficios que le ofrezca.
En la estructuración de las preguntas del cuestionario aplicado se tuvo en cuenta dos factores importantes relacionados con los obsequios, los cuales constituyen una guía de la forma en que se deben enfocar los esfuerzos en este aspecto de la compra: 1) Evaluar la influencia que tuvo este en la decisión final de compra y 2) Evaluar la efectividad de algunos obsequios que actualmente se están dando y otros que se podrían dar.

De los resultados de la Tabla 2 se puede observar que las opiniones con respecto al nivel de influencia están divididas, dado que el $51,7 \%$ de las personas afirmaron que el obsequio o fue influyente o muy influyente en su decisión de compra, mientras que un $48,3 \%$ opinaron que este aspecto fue o indiferente o poco influyente o muy poco influyente o NS/NR (no saben / no responden). Estos resultados pueden ser un indicador de irrelevancia de ofrecer obsequios por la compra, y de que se deben considerar otras estrategias enfocadas en otros aspectos. 
Tabla 2. Compilación de preguntas relacionadas con obsequios

Table 2. Compilation of questions concerning gifts

\begin{tabular}{lc}
\hline \multicolumn{2}{|c}{ ¿Con la compra realizada se le ofreció algún servicio u obsequio adicional? } \\
\hline Sí & $55 \%$ \\
\hline No & $45 \%$ \\
\hline \multicolumn{2}{|c|}{ Señale el nivel de influencia que tuvo el obsequio que le ofrecieron al momento } \\
$\quad$ de tomar la decisión de comprar la motocicleta
\end{tabular}

Fuente: Elaboración propia

Dentro de la calificación de los obsequios que se dan y que se podrían dar, se obtuvo para cada uno de ellos una calificación con escala tipo Likert de 5 niveles (muy importante, importante, indiferente, poco importante y nada importante). Cada encuestado tuvo que calificar cada uno de estos artículos con un valor de la escala. Los resultados se muestran en la Tabla 3:

Tabla 3. Calificación de los diferentes obsequios ofrecidos

Table 3. Qualification of different gifts offered

Importancia de los posibles obsequios

\begin{tabular}{lccccc}
\hline & Muy importante & Importante & Indiferente & $\begin{array}{c}\text { Poco } \\
\text { importante }\end{array}$ & $\begin{array}{c}\text { Nada } \\
\text { importante }\end{array}$ \\
\hline Marcación de la motocicleta & $48,0 \%$ & $42,0 \%$ & $5,0 \%$ & $5,0 \%$ & $0,0 \%$ \\
\hline Una mochila & $12,0 \%$ & $22,0 \%$ & $38,0 \%$ & $15,0 \%$ & $13,0 \%$ \\
\hline $\begin{array}{l}\text { Artículos de su equipo de } \\
\text { fútbol preferido }\end{array}$ & $5,0 \%$ & $28,0 \%$ & $28,0 \%$ & $14,0 \%$ & $25,0 \%$ \\
\hline Bonos de gasolina & $22,0 \%$ & $57,0 \%$ & $13,0 \%$ & $5,0 \%$ & $3,0 \%$ \\
\hline Un segundo casco & $47,0 \%$ & $33,0 \%$ & $15,0 \%$ & $5,0 \%$ & $0,0 \%$ \\
\hline Descuentos en accesorios & $32,0 \%$ & $40,0 \%$ & $22,0 \%$ & $6,0 \%$ & $0,0 \%$ \\
\hline $\begin{array}{l}\text { Un impermeable } \\
\text { Carnet preferencial para obtener }\end{array}$ & $35,0 \%$ & $47,0 \%$ & $10,0 \%$ & $5,0 \%$ & $3,0 \%$ \\
descuentos en repuestos & $35,0 \%$ & $45,0 \%$ & $15,0 \%$ & $5,0 \%$ & $0,0 \%$
\end{tabular}

Qué tan de acuerdo o en desacuerdo está con la siguiente afirmación: El obsequio que se ofrece por el concesionario influenció fuertemente mi decisión de compra

\begin{tabular}{cccccc|}
\hline Totalmente de acuerdo & $\begin{array}{c}\text { Medianamente } \\
\text { de acuerdo }\end{array}$ & Indiferente & $\begin{array}{c}\text { Medianamente } \\
\text { en desacuerdo }\end{array}$ & $\begin{array}{c}\text { Totalmente } \\
\text { en } \\
\text { desacuerdo }\end{array}$ & $\begin{array}{c}\text { No sabe/ No } \\
\text { responde }\end{array}$ \\
\hline $13,30 \%$ & $41,7 \%$ & $30,0 \%$ & $5,0 \%$ & $10,0 \%$ & $0,0 \%$ \\
\hline
\end{tabular}

Fuente: elaboración propia. 
Para obtener una jerarquización de los obsequios, se realizó un proceso de calificación por puntos en donde las categorías «muy importante» e «importante» tenían puntos positivos proporcionales a su grado de influencia, y las categorías «poco importante» y «nada importante» tenían puntos negativos. Tras la ponderación se obtuvo la siguiente jerarquización:

Tabla 4. Jerarquización de los obsequios

Table 4. Hierarchy of gifts

\begin{tabular}{lc}
\hline Obsequio & Peso \\
\hline Marcación de la motocicleta & 4,13 \\
\hline Un segundo casco & 4,02 \\
\hline Carnet preferencial para obtener descuentos en repuestos & 3,9 \\
\hline Un impermeable & 3,72 \\
\hline Descuentos en accesorios & 3,7 \\
\hline Bonos de gasolina & 3,55 \\
\hline Una mochila & 1,9 \\
\hline Artículos de su equipo de fútbol preferido & 1,22 \\
\hline
\end{tabular}

Dentro de la jerarquización, inicialmente se supuso que las personas prefieren uno u otro obsequio, según el precio que tienen comercialmente, pero al observar los resultados se infiere que existen otros factores como la duración del artículo, así como su aporte a la funcionalidad y seguridad de la moto, destacándose como aspectos que influyen en la calificación del obsequio. Un ejemplo de ello son los bonos de gasolina, los cuales tienen un valor igual o superior que la marcación de la moto, pero por el hecho de que estos bonos se consumen más rápidamente que la marcación, entonces la sensación de que se obtuvo algo gratis va a ser menos duradera. Por otro lado, un segundo casco posee durabilidad, aporta a la seguridad y es una necesidad básica de un motociclista; razones por los cuales pudo haber recibido una de las puntuaciones más altas, y no fue solo por su valor comercial.

De esta caracterización de los obsequios se podría concluir que al generar estrategias para incrementar las ventas a través de estos, lo más importante es que sea algo que perdure, que tenga funcionalidad complementaria con la motocicleta y que aporte a la seguridad o a la comodidad.

\section{Influencia de terceros}

Chadha et al. (2011) considera la influencia de terceros como el factor más impactante en las decisiones del consumidor de motocicletas. Para este estudio este factor hace referencia a cualquier institución, persona o medio que el cliente consultó para realizar su compra. En la Tabla 5 se presentan las preguntas de la encuesta que miden este factor $y$ el porcentaje de encuestados que eligió cada una de las posibles respuestas.

De la Tabla 5 se puede observar que el factor «influencia de terceros» mostró los siguientes resultados:

- El cliente, al momento de seleccionar el tipo de motocicleta, tuvo en cuenta la recomendación de un(a) amigo(a) (58,3\%), de un familiar $(48,3 \%)$ o incluso de la publicidad vía internet $(41,7 \%)$, antes que por redes sociales $(18,3 \%)$ o un(a) asesor(a) de ventas $(18,3 \%)$.

- La mayoría de los clientes admiten que la publicidad es influyente (35\%) o muy influyente (37\%) en la decisión de compra de la moto. 
La mayoría de los clientes tienen potenciales referidos $(68,3 \%)$ o estarían de acuerdo en traer referidos a cambio de algún obsequio $(81,7 \%)$ o con el fin de acceder a un beneficio para ellos.

Estos resultados evidencian una importante oportunidad para ser explorada por la gerencia del concesionario, ya que, un alto porcentaje de clientes estarían dispuestos a llevar referidos a este, los cuales también tendrían posibles referidos. Esto significa que los mismos clientes del concesionario tienen un potencial significativo de constituirse en un canal para mejorar el posicionamiento del mismo en el mercado y atraer nuevos compradores. Esto se ratifica en el alto porcentaje de personas que respondieron que habían sabido del concesionario por parte de un conocido.

Tabla 5. Influencia de terceros

Table 5. Influence by third parties

6. ¿Por cuáles de las siguientes razones escogió el lugar de compra de su motocicleta? (Selección múltiple).

Opción 6.5. Un conocido le hizo una recomendación.

$28,30 \%$

14. Al momento de hacer la compra de la moto pidió consejo a (se pueden seleccionar varias opciones):

\begin{tabular}{|c|c|c|c|c|c|c|c|}
\hline Amigo(a) & Familiar & Asesor(a) & Red Social & Internet & Foros & Videos & $\begin{array}{c}\text { No pidió ningún } \\
\text { consejo }\end{array}$ \\
\hline $58,30 \%$ & $48,30 \%$ & $18,30 \%$ & $18,30 \%$ & $41,70 \%$ & $18,30 \%$ & $13,30 \%$ & $6,70 \%$ \\
\hline \multicolumn{8}{|c|}{ 15. Influencia de la publicidad en la decisión de compra. } \\
\hline \multicolumn{3}{|l|}{ Muy influyente } & \multicolumn{2}{|c|}{ Indiferente } & \multicolumn{2}{|c|}{ Poco influyente } & Muy poco influyente \\
\hline \multicolumn{2}{|l|}{$16,70 \%$} & $55,00 \%$ & \multicolumn{2}{|c|}{$8,30 \%$} & \multicolumn{2}{|c|}{$8,30 \%$} & $11,70 \%$ \\
\hline \multicolumn{8}{|c|}{ 16. ¿Traería referidos a cambio de algún obsequio para usted? } \\
\hline \multicolumn{4}{|c|}{ Sí } & \multicolumn{4}{|c|}{ No } \\
\hline \multicolumn{4}{|c|}{$81,70 \%$} & \multicolumn{4}{|c|}{$18,30 \%$} \\
\hline \multicolumn{8}{|c|}{ 17. ¿Tiene potenciales referidos? } \\
\hline \multicolumn{4}{|c|}{ Sí } & \multicolumn{4}{|c|}{ No } \\
\hline \multicolumn{4}{|c|}{$68,30 \%$} & \multicolumn{4}{|c|}{$31,70 \%$} \\
\hline
\end{tabular}

18.6. Influencia de las recomendaciones.

$\begin{array}{ccccc}\text { Muy influyente } & \text { Influyente } & \text { Indiferente } & \text { Poco influyente } & \text { Muy poco influyente } \\ 37,00 \% & 35,00 \% & 16,00 \% & 10,00 \% & 2,00 \%\end{array}$

21. ¿Cómo se enteró de la existencia del concesionario? (se pueden seleccionar varias opciones)

\begin{tabular}{ccccc}
$\begin{array}{c}\text { 21.1. Referido por } \\
\text { un conocido }\end{array}$ & $\begin{array}{c}\text { 21.2. Publicidad en } \\
\text { televisión }\end{array}$ & $\begin{array}{c}\text { 21.3. Publicidad en } \\
\text { periódicos }\end{array}$ & $\begin{array}{c}\text { 21.4. Alguien } \\
\text { conocido trabaja allí }\end{array}$ & 21.5. Redes sociales \\
\hline $68,30 \%$ & $25,00 \%$ & $13,30 \%$ & $15,00 \%$ & $10,00 \%$
\end{tabular}

Fuente: Elaboración propia 
Análisis cruzado

Influencia de terceros
Tabla 6. Análisis cruzado influencia de terceros

Table 6. Cross-analysis of the Influence by third parties

\begin{tabular}{cccc}
\hline \multirow{2}{*}{ ¿Tiene potenciales referidos? } & \multicolumn{3}{c}{ ¿Traería referidos a cambio de un obsequio? } \\
\cline { 2 - 4 } & No & Sí & Total general \\
\hline No & $8,33 \%$ & $23,33 \%$ & $31,67 \%$ \\
\hline Sí & $10,00 \%$ & $58,33 \%$ & $68,33 \%$ \\
\hline Total general & $18,33 \%$ & $81,67 \%$ & $100,00 \%$ \\
\hline
\end{tabular}

Fuente: Elaboración propia.

En la Tabla 6 se muestra que el $81,67 \%$ de los clientes, sin importar que tengan o no tengan referidos, llevarían referidos a cambio de un obsequio. Si se relacionan estos datos con el análisis de la Tabla 2 que indica que finalmente un obsequio no es determinante para definir la realización o no de la compra, se podría concluir que los obsequios tendrían un mayor impacto sobre las ventas si son ofrecidos a los clientes que lleven a otros compradores al concesionario, en lugar de entregarlos directamente al cliente como incentivo para la compra.

En este sentido, la gerencia del concesionario podría entonces constituir una buena estrategia de ventas incentivando a sus clientes por medio de obsequios para que traigan otros compradores (política de referidos).

Proceso de compra

Tabla 7. Análisis cruzado del proceso de compra

Table 7. Cross-analysis of the buying process

\begin{tabular}{|c|c|c|c|c|c|}
\hline \multirow{2}{*}{\multicolumn{2}{|c|}{$\begin{array}{l}\text { Periodo de tiempo entre la cotización } \\
\qquad \text { y la compra }\end{array}$}} & \multicolumn{4}{|c|}{ ¿Tenía claro que motocicleta adquirir? } \\
\hline & & \multicolumn{2}{|c|}{ No } & \multicolumn{2}{|l|}{ Sí } \\
\hline \multicolumn{2}{|c|}{ De 0 a 2 semanas } & \multicolumn{2}{|c|}{$13,33 \%$} & \multicolumn{2}{|l|}{$17,78 \%$} \\
\hline \multicolumn{2}{|c|}{ De 2 a 4 semana } & \multicolumn{2}{|c|}{$53,34 \%$} & \multicolumn{2}{|l|}{$53,33 \%$} \\
\hline \multicolumn{2}{|c|}{ De 4 a 6 semanas } & \multicolumn{2}{|c|}{$33,33 \%$} & \multicolumn{2}{|l|}{$17,78 \%$} \\
\hline \multicolumn{2}{|c|}{ De 6 semanas - 2 meses } & \multicolumn{2}{|c|}{$0,00 \%$} & \multicolumn{2}{|l|}{$8,89 \%$} \\
\hline \multicolumn{2}{|c|}{ Más de 2 meses } & \multicolumn{2}{|c|}{$0,00 \%$} & \multicolumn{2}{|l|}{$2,22 \%$} \\
\hline \multicolumn{2}{|c|}{ Total general } & & & \multicolumn{2}{|l|}{$100,00 \%$} \\
\hline \multirow{2}{*}{$\begin{array}{l}\text { ¿Tenía claro } \\
\text { qué } \\
\text { motocicleta } \\
\text { adquirir? }\end{array}$} & \multicolumn{5}{|c|}{$\begin{array}{l}\text { ¿La amabilidad del asesor, el grado de empatía que posea y la actitud de colaboración son factores } \\
\text { decisivos en el momento de escoger en qué concesionario se compra la moto? }\end{array}$} \\
\hline & $\begin{array}{l}\text { Medianamente } \\
\text { en desacuerdo }\end{array}$ & Indiferente & $\begin{array}{l}\text { Medianamente } \\
\text { de acuerdo }\end{array}$ & $\begin{array}{l}\text { Totalmente de } \\
\text { acuerdo }\end{array}$ & $\begin{array}{l}\text { Total } \\
\text { general }\end{array}$ \\
\hline No & $6,67 \%$ & $20,00 \%$ & $53,33 \%$ & $20,00 \%$ & $100 \%$ \\
\hline Sí & $0,00 \%$ & $6,67 \%$ & $51,11 \%$ & $42,22 \%$ & $100 \%$ \\
\hline
\end{tabular}

Fuente: Elaboración propia. 
Frente al porcentaje de personas que demoran más que el promedio en decidirse a comprar la motocicleta (Tabla 7), se enfatiza que es mayor en las personas que no tienen claro qué modelo comprar al momento de llegar al concesionario. Sin embargo, los mayores lapsos de tiempo para llevar a cabo la compra se dan en los clientes que tienen claro qué modelo desean adquirir.

También se aprecia que aunque los compradores tuvieran o no tuvieran claro qué motocicleta comprar, ellos valoran en buena medida la calidad de la atención que se les ofrece, ya que un 93,33\% de los clientes que tenían claro qué motocicleta comprar desde el inicio y un $73,3 \%$ de los que no lo tenían claro, afirmaron estar «medianamente de acuerdo» o «totalmente de acuerdo» en que la calidad de la atención es un factor decisivo en el momento de escoger en qué concesionario se compra la moto.

\section{CONCLUSIONES}

Con el análisis de resultados de la investigación, se concluye que una de las situaciones más importantes a evaluar tiene que ver con las formas de relacionar la influencia de los obsequios con la influencia de terceros. En este aspecto, se encontró que los clientes valoran positivamente que se les ofrezca algún obsequio, aunque igualmente se encontró que al momento de la compra esto no fue determinante.

Por otra parte, los encuestados no solo manifestaron un alto interés en llevar nuevos compradores a cambio de obsequios sino también, que de hecho, tenían posibles referidos y que ellos mismos en gran medida habían tenido conocimiento del concesionario por medio de un conocido.

El análisis de resultados mostró también que los asesores de ventas no tienen tanta influencia en las ventas como lo tiene un familiar o una persona con lazos más cercanos al cliente. Estos resultados se hacen más interesantes aún si se contrastan con el hecho de que una proporción significativamente alta de los encuestados afirmaron que la calidad de atención por parte del asesor es un factor definitivo, lo cual es un elemento importante que se debe considerar en el intento de reducir los tiempos de compra.

De acuerdo con la caracterización de las personas encuestadas, se tiene que el grueso de los individuos son personas de mediana edad con ingresos mensuales inferiores al $\$ 1^{\prime} 800.000$, lo cual es un elemento a tener en cuenta al dirigir las estrategias de ventas y financiamiento de acuerdo con la capacidad de compra de los clientes del concesionario, lo cual incluye por ejemplo consideraciones del tipo de incluir líneas de microcrédito para los clientes de tal modo que llegue a más cantidad de personas y con esto se pueda aumentar la cantidad de ventas en el concesionario.

Como recomendación para el concesionario se encuentra la de llevar a cabo una capacitación que permita a los asesores generar altos niveles de calidad en la atención y tener más empatía con el cliente, para aumentar las ventas y lograr una disminución de tiempo en el proceso de compra. Sin embargo, en términos de futuras investigaciones, se recomienda indagar más detalladamente por el hecho, de que si bien, los compradores tienen en menor consideración las recomendaciones de vendedores 0 representantes de la compañía, también afirman que la empatía con estos es un factor definitivo en la compra.

Finalmente se aconseja que en próximos estudios se desagregue el factor «influencia de terceros» en más factores, de forma que se logre diferenciar entre la percepción que tienen los compradores de las sugerencias o recomendaciones que hacen asesores o representantes de la empresa, de las que les hacen sus propios conocidos, ya que en otras investigaciones de este tipo se encontraron resultados interesantes al diferenciar estos elementos. 


\section{REFERENCIAS}

Ariely, D., \& Berns, G. S. (2010). Neuromarketing: the hope and hype of neuroimaging in business. Nature reviews. Neuroscience, 11(4), 284-92. doi:10.1038/nrn2795

Chadha, O. (2011). Study Of Consumer Buying Behavior Towards Bikes. International Journal of Research in Finance \& Marketing, 1(2), 86101.

Comité de Ensambladoras de Motos Japonesas. (2013). VIII Estudio sociodemográfico del usuario de la moto en colombia, 8 .

Fedorikhin, A. (1999). Heart and Mind in Conflict : The Interplay of Affect and Cognition in Consumer Decision Making. Journal of Consumer Research, 26(3), 278-292.

Fenalco Antioquia. (2013). Boletín Económico Sectorial- Sector Motos, (19), 1-23. Retrieved from http://www.andi.com.co/pages/noticias/notic ia_detalle.aspx? IdNews=369.

Hair, J. F., Bush, R. P., \& Ortinau, D. J. (2010). Investigación de mercados. McGraw-Hill.

Khan, M. S. K., Jain, M. V., \& Sharma, M. A. (2013). The Effect Of Brand Positioning On Customer Buying Decisions And Brand Loyalty Towards Deluxe Segment of Motorcycle With Reference To India Yamaha Motors. International Journal of Research in Management \& IT, 1(1), 175-184.

Khan, S. G. N., Siddiqui, J., Shah, B. A., \& Hunjra, A. I. (2012). Effective advertising and its influence on consumer buying behavior. Information Management and Business Review, 4(3), 114119.

Kathiravanaa, C., Panchanathamaa, N., \& Anushan, S. (2010). The Competitive Implications Of Consumer Evaluation Of Brand Image, Product Attributes, And Perceived Quality In Competitive Two-Wheeler Markets Of India. Serbian Journal of Management, 5(1), 21-38.

Lai, C.-S. (2007). The effects of influence strategies on dealer satisfaction and performance in
Taiwan's motor industry. Industrial Marketing Management, 36(4), 518-527. doi:10.1016/j.indmarman.2005.08.015

Laudon K. C. \& Traver C. G. 2011. E-Commerce 2011. 7th ed. Pearson Education: England.

Lefkoff-hagius, R., \& Mason, C. H. (1993). Characteristic, Beneficial, and Image Attributes in Consumer Judgments of Similarity and Preference. Journal of Consumer Research, 20, 100-111.

Lomboan, R., Saerang, D. P. E., \& Pangemanan, S. S. (2013). The Influence Of Motivation And Perception As Psychological Factors On Consumer Buying Decision Of Kawasaki Ninja Motorcycle. Jurnal EMBA, 1(4), 2354-2361. Retrieved from http://scholar.google.com/scholar?hl=en\&btn $\mathrm{G}=$ Search\&q=intitle:No+Title\#0

McDaniel, C., \& Gates, R. (2007). Marketing research essential.

Ministerio de Comercio Industria y Turismo República de Colombia (2013). Informe de industria (pp. 1-36).

Ngantung, V. N. (2013). Determinant factors of product attributes to consumer buying behavior of motorcycles. Jurnal EMBA, 1(4), 697-707.

Pinch, P., \& Reimer, S. (2010). Moto-mobilities: geographies of the motorcycle and motorcyclists Moto-mobilities : geographies of the motorcycle and motorcyclists Abstract, 44(0), 1-32.

Pride W. M., Hughes R. J., \& Kapoor J. R. 2010. Business. Disponible en http://college.cengage.com/busines/course3 60/intro_to_business_1111101213/Pride_03 24829558_ch02-pdf.

Putthiwanit, C. (2012). Investigating Consumer Insight by Using Completion Techniques: A Pilot Study of a Motorcycle Accessory Shop in Thailand. International Review of Management and Marketing, 2(2), 92-98. 
Reddy, D. K. M. (2011). Consumers Behaviour Towards Two-Wheeler Motor Bikes. Osmania journal of Management, 1(1), 1-9.

Sekaran, U., \& Bougie, R. (2009). Research methods of business: A skill-building approach (ed.). New York: John Willey \& Sons.

Srinivas, S. (2011). Consumer satisfaction on two wheeler motor bikes: a study on nandyal, kurnool district, a.p., india. International journal of research in commerce \& management, 2(3), 125-134.

Solomon M. R. 2011. Consumer Behavior: Buying, Having, and Being. 9th ed. Pearson Education: New Jersey.

Uddin, A. (2012). Factors Responsible to Dealers' Satisfaction: A Study on Partex Furniture Industries Ltd. World Journal of Social Sciences, 2(5), 207-217.

Varadarajan, R. (2009). Strategic marketing and marketing strategy: domain, definition, fundamental issues and foundational premises. Journal of the Academy of Marketing Science, 38(2), 119-140. doi:10.1007/s11747009-0176-7

Wu, P. C. S., Yeh, G. Y.-Y., \& Hsiao, C.-R. (2011). The effect of store image and service quality on brand image and purchase intention for private label brands. Australasian Marketing Journal (AMJ), 19(1), 30-39. doi:10.1016/j.ausmj.2010.11.001

Yuvaraju, D. (2014). Customer Satisfaction Towards TVS Apache: A Case Study in Tirupati. International Journal of Commerce, Business and Management, 3(3), 379-394.

Zahay, D., \& Griffin, A. (2010). Marketing strategy selection, marketing metrics, and firm performance. Journal of Business \& Industrial Marketing, 25(2), 84-93. doi:10.1108/08858621011017714 